\title{
Existence in the Large of Periodic Solutions of Hyperbolic Partial Differential Equations
}

\section{LAMBerto Cesari}

The problem of existence of solutions $\varphi(x, y)$ periodic in $x$ and in $y$ of period $T$ for a hyperbolic partial differential system of the form

$$
u_{x y}=f\left(x, y, u, u_{x}, u_{y}\right)
$$

where $u=\left(u_{1}, \ldots, u_{n}\right)$, and $f=\left(f_{1}, \ldots, f_{n}\right)$ is periodic in $x$ and $y$ of period $T$, presents a number of difficulties when no damping of any sort is assumed. In this paper we analyze this difficult problem in the line of our previous work on ordinary and partial differential equations. We conclude with criteria of existence for solutions to the problem above. These criteria can then be used for the analogous problem for the equation

$$
u_{x x}-u_{y y}=g\left(x, y, u, u_{x}, u_{y}\right)
$$

\section{The Modified Problem}

1. Modified Problem. We shall first associate with (1) the following analogous weaker problem or modified problem:

Given two periodic functions $u_{0}(x), v_{0}(y)$ of class $C^{1}$ in $(-\infty,+\infty)$ and of period $T$,

$$
u_{0}(x+T)=u_{0}(x), \quad v_{0}(x+T)=v_{0}(x),
$$

determine a function $\Phi(x, y)$ continuous with its partial derivatives $\Phi_{x}, \Phi_{y}, \Phi_{x y}$, two functions $m(y)$ and $n(x)$ both continuous, and a constant $\mu$, such that

$$
\begin{gathered}
\Phi(x+T, y)=\Phi(x, y)=\Phi(x, y+T), \quad m(x+T)=m(x), \quad n(y+T)=n(y), \\
\int_{0}^{T} m(y) d y=0, \quad \int_{0}^{T} n(x) d x=0,
\end{gathered}
$$

and

$$
\Phi_{x y}=f\left(x, y, \Phi, \Phi_{x}, \Phi_{y}\right)-m(y)-n(x)-\mu .
$$

For this modified problem we shall prove theorems of existence, uniqueness, and continuous dependence on the boundary values and parameters. In (4) we assume

$$
f(x+T, y, z, p, q)=f(x, y, z, p, q)=f(x, y+T, z, p, q) .
$$

Then the function $\Phi$ is a periodic solution of the original problem (1) if and only if we can determine $u_{0}(x), v_{0}(y)$ in such a way that

$$
\mu=0, \quad m(y) \equiv 0, \quad n(x) \equiv 0 .
$$

Criteria for this occurrence are given in Sections 12-19. 
2. Theorem I (existence theorem for the modified problem). If $T>0$, and $N, N_{1}, N_{2}, L, b_{1}, b_{2}, M_{1}, M_{2}, M_{3} \geqq 0$ are constants, if $A$ and $R$ are the sets

$$
\begin{aligned}
& A=[0 \leqq x \leqq T, 0 \leqq y \leqq T], \\
& R=\left[0 \leqq x \leqq T, 0 \leqq y \leqq T,|z| \leqq M_{1},|p| \leqq M_{2},|q| \leqq M_{3}\right],
\end{aligned}
$$

if if

$$
M_{1} \geqq N+\left(N_{1}+N_{2}\right) T / 2+3 L T^{2}, \quad M_{2} \geqq N_{1}+3 L T, \quad M_{3} \geqq N_{2}+3 L T,
$$

$$
u_{0}(x), \quad 0 \leqq x \leqq T, \quad v_{0}(y), \quad 0 \leqq y \leqq T,
$$

are vector functions which are continuous with $u_{0}^{\prime}(x), v_{0}^{\prime}(y)$, if

$$
f(x, y, z, p, q), \quad(x, y, z, p, q) \in R,
$$

is continuous in $R$, and

$$
\begin{gathered}
u_{0}(T)=u_{0}(0), \quad v_{0}(T)=v_{0}(0)=0, \quad u_{0}^{\prime}(T)=u_{0}^{\prime}(0), \quad v_{0}^{\prime}(T)=v_{0}^{\prime}(0) \\
\left|u_{0}(0)\right| \leqq N,\left|u_{0}\left(x_{1}\right)-u_{0}\left(x_{2}\right)\right| \leqq N_{1}\left|x_{1}-x_{2}\right|,\left|v_{0}\left(y_{1}\right)-v_{0}\left(y_{2}\right)\right| \leqq N_{2}\left|y_{1}-y_{2}\right| \\
f(T, y, z, p, q)=f(0, y, z, p, q), \quad f(x, T, z, p, q)=f(x, 0, z, p, q) \\
|f(x, y, z, p, q)| \leqq L \\
\left|f\left(x, y, z, p_{1}, q_{1}\right)-f\left(x, y, z, p_{2}, q_{2}\right)\right| \leqq b_{1}\left|p_{1}-p_{2}\right|+b_{2}\left|q_{1}-q_{2}\right|
\end{gathered}
$$

then for

$$
2 T b_{1}<1, \quad 2 T b_{2}<1,
$$

there exist a vector function $\varphi(x, y),(x, y) \in A$, continuous in $A$ together with $\varphi_{x}, \varphi_{y}, \varphi_{x y}$, continuous vector functions $m(y), 0 \leqq y \leqq T, n(x), 0 \leqq x \leqq T$, and $a$ constant $\mu$, such that

$$
\begin{gathered}
\varphi(x, 0)=\varphi(x, T)=u_{0}(x), \quad \varphi_{y}(x, 0)=\varphi_{y}(x, T), \\
\varphi(0, y)=\varphi(T, y)=u_{0}(0)+v_{0}(y), \quad \varphi_{x}(0, y)=\varphi_{x}(T, y), \\
m(0)=m(T), \quad n(0)=n(T), \\
\varphi_{x y}(x, y)=f\left(x, y, \varphi(x, y), \varphi_{x}(x, y), \varphi_{y}(x, y)\right)-m(y)-n(x)-\mu, \\
\int_{0}^{T} m(\eta) d \eta=0, \quad \int_{0}^{T} n(\xi) d \xi=0, \\
\mu=T^{-2} \int_{0}^{T} \int_{0}^{T} f\left(\xi, \eta, \varphi(\xi, \eta), \varphi_{x}(\xi, \eta), \varphi_{y}(\xi, \eta)\right) d \xi d \eta, \\
m(y)=T^{-1} \int_{0}^{T} f\left(\xi, y, \varphi(\xi, y), \varphi_{x}(\xi, y), \varphi_{y}(\xi, y)\right) d \xi-\mu, \\
n(x)=T^{-1} \int_{0}^{T} f\left(x, \eta, \varphi(x, \eta), \varphi_{x}(x, \eta), \varphi_{y}(x, \eta)\right) d \eta-\mu,
\end{gathered}
$$


for all $0 \leqq x \leqq T, 0 \leqq y \leqq T$. Thus, by extending all functions $\varphi(x, y), m(y), n(x)$, $f(x, y, z, p, q)$ for all $-\infty<x, y<+\infty,|z| \leqq M_{1},|p| \leqq M_{2},|q| \leqq M_{3}$, by means of the periodicity of period $T$ in $x$ and $y$, equation (14) is satisfied in the whole $x y$-plane.

3. Proof of Theorem 1. First let us note that relations (11), (12), (14), (15), imply (16), (17), (18). Indeed, by integration of (14) on $A$, we deduce (16). Then, by integration of (14) again on $0 \leqq x \leqq T$, or on $0 \leqq y \leqq T$, we deduce (17) and (18), respectively. Note that (8), (11), (12), (17), (18) imply (13), and that (16), (17), (18) imply (15).

Let us first prove that every vector function $\varphi(x, y),(x, y) \in A$, satisfying

$$
\begin{aligned}
& \varphi(x, 0)=\varphi(x, T)=u_{0}(x), \quad \varphi(0, y)=\varphi(T, y)=u_{0}(0)+v_{0}(y), \\
& \left|\varphi\left(x_{1}, y_{1}\right)-\varphi\left(x_{1}, y_{2}\right)-\varphi\left(x_{2}, y_{1}\right)+\varphi\left(x_{2}, y_{2}\right)\right| \leqq 6 L\left|x_{1}-x_{2}\right|\left|y_{1}-y_{2}\right|,
\end{aligned}
$$

also satisfies the relations

$$
\begin{gathered}
|\varphi(x, y)| \leqq M_{1}, \quad\left|\varphi\left(x_{1}, y\right)-\varphi\left(x_{2}, y\right)\right| \leqq M_{2}\left|x_{1}-x_{2}\right|, \\
\left|\varphi\left(x, y_{1}\right)-\varphi\left(x, y_{2}\right) \leqq M_{3}\right| y_{1}-y_{2} \mid .
\end{gathered}
$$

Indeed, we have, for $0 \leqq x \leqq T, 0 \leqq y \leqq T$,

where

$$
\left|\varphi\left(x_{1}, y\right)-\varphi\left(x_{2}, y\right)-\varphi\left(x_{1}, 0\right)+\varphi\left(x_{2}, 0\right)\right| \leqq 6 L\left|x_{1}-x_{2}\right| y
$$

and hence

$$
\left|\varphi\left(x_{1}, 0\right)-\varphi\left(x_{2}, 0\right)\right|=\left|u_{0}\left(x_{1}\right)-u_{0}\left(x_{2}\right)\right| \leqq N_{1}\left|x_{1}-x_{2}\right|,
$$

$$
\left|\varphi\left(x_{1}, y\right)-\varphi\left(x_{2}, y\right)\right| \leqq\left(N_{1}+6 L y\right)\left|x_{1}-x_{2}\right| .
$$

Analogously, we have

$$
\left|\varphi\left(x_{1}, y\right)-\varphi\left(x_{2}, y\right)\right| \leqq\left(N_{1}+6 L(T-y)\right)\left|x_{1}-x_{2}\right| .
$$

Since either $0 \leqq y \leqq T / 2$ or $0 \leqq T-y \leqq T / 2$, we have

$$
\left|\varphi\left(x_{1}, y\right)-\varphi\left(x_{2}, y\right)\right| \leqq\left(N_{1}+3 L T\right)\left|x_{1}-x_{2}\right| \leqq M_{2}\left|x_{1}-x_{2}\right| .
$$

Analogously, we prove that

$$
\left|\varphi\left(x, y_{1}\right)-\varphi\left(x, y_{2}\right)\right| \leqq\left(N_{2}+3 L T\right)\left|y_{1}-y_{2}\right| \leqq M_{3}\left|y_{1}-y_{2}\right| .
$$

Finally, for $0 \leqq x, y \leqq T / 2$,

$$
\begin{aligned}
|\varphi(x, y)| & \leqq|\varphi(0,0)|+|\varphi(0, y)-\varphi(0,0)|+|\varphi(x, y)-\varphi(0, y)| \\
& \leqq N+N_{2} y+\left(N_{1}+6 L y\right) x \leqq N+\left(N_{1}+N_{2}\right) T / 2+3 L T^{2} \leqq M_{1} .
\end{aligned}
$$

Analogous reasoning holds for $(x, y)$ in the remaining quadrants of $A$. Thus $|\varphi(x, y)| \leqq M_{1},(x, y) \in A$. We have proved that relations (19) imply (20). Also, the vector functions $\varphi(x, y)$ satisfying (20) are all Lipschitzian in $A$, and hence have partial derivatives $\varphi_{x}, \varphi_{y}$ a.e. in $A$ satisfying $\left|\varphi_{x}\right| \leqq M_{2},\left|\varphi_{y}\right| \leqq M_{3}$ a.e. in $A$. 
The vector function $f(x, y, z, p, q)$ is continuous in $R$. Hence, there are continuous monotone functions $\omega_{1}(\alpha), \omega_{2}(\beta), \omega_{3}(\gamma)$ in $[0,+\infty)$ such that $\omega_{1}(0)=$ $\omega_{2}(0)=\omega_{3}(0)=0$, and

for all

$$
\begin{aligned}
& \left|f\left(x_{1}, y, z, p, q\right)-f\left(x_{2}, y, z, p, q\right)\right| \leqq \omega_{1}\left(\left|x_{1}-x_{2}\right|\right), \\
& \left|f\left(x, y_{1}, \dot{2}, p, q\right)-f\left(x, y_{2}, z, p, q\right)\right| \leqq \omega_{2}\left(\left|y_{1}-y_{2}\right|\right), \\
& \left|f\left(x, y, z_{1}, p, q\right)-f\left(x, y, z_{2}, p, q\right)\right| \leqq \omega_{3}\left(\left|z_{1}-z_{2}\right|\right),
\end{aligned}
$$

$0 \leqq x, x_{1}, x_{2}, y, y_{1}, y_{2} \leqq T, \quad|z|,\left|z_{1}\right|,\left|z_{2}\right| \leqq M_{1}, \quad|p| \leqq M_{2}, \quad|q| \leqq M_{3}$.

The vector functions $u_{0}^{\prime}(x), v_{0}^{\prime}(y)$ are continuous in $[0, T]$. Hence, there are continuous monotone functions $\omega_{4}(\alpha), \omega_{5}(\beta), 0 \leqq \alpha, \beta<+\infty$, with $\omega_{4}(0)=\omega_{5}(0)$ $=0$, such that

Let

$$
\left|u_{0}^{\prime}\left(x_{1}\right)-u_{0}^{\prime}\left(x_{2}\right)\right| \leqq \omega_{4}\left(\left|x_{1}-x_{2}\right|\right), \quad\left|v_{0}^{\prime}\left(y_{1}\right)-v_{0}^{\prime}\left(y_{2}\right)\right| \leqq \omega_{5}\left(\left|y_{1}-y_{2}\right|\right) .
$$

$$
\begin{aligned}
& \eta_{1}(\beta)=\left(1-2 T b_{2}\right)^{-1}\left[\omega_{5}(\beta)+2 T \omega_{2}(\beta)+2 T \omega_{3}\left(M_{3} \beta\right)+12 L T b_{1} \beta\right], \\
& \eta_{2}(\alpha)=\left(1-2 T b_{1}\right)^{-1}\left[\omega_{4}(\alpha)+2 T \omega_{1}(\alpha)+2 T \omega_{3}\left(M_{2} \alpha\right)+12 L T b_{2} \alpha\right] .
\end{aligned}
$$

Both $\eta_{1}(\beta)$ and $\eta_{2}(\alpha), 0 \leqq \alpha, \beta<+\infty$, are continuous monotone functions with $\eta_{1}(0)=\eta_{2}(0)=0$.

Let $E$ be the linear space of all vector functions $\varphi(x, y),(x, y) \in A$, continuous in $A$ together with their partial derivatives $\varphi_{x}, \varphi_{y}$ with norm $\|\varphi\|=\max |\varphi|+$ $\max \left|\varphi_{x}\right|+\max \left|\varphi_{y}\right|$, where max is taken in $A$.

Let $K$ be the subset of $E$ made up of all vector functions $\varphi(x, y) \in E$ satisfying relations (19) and in addition

$$
\begin{gathered}
\varphi_{x}(0, y)=\varphi_{x}(T, y), \quad \varphi_{y}(x, 0)=\varphi_{y}(x, T), \\
\left|\varphi_{x}\left(x_{1}, y\right)-\varphi_{x}\left(x_{2}, y\right)\right| \leqq \eta_{2}\left(\left|x_{1}-x_{2}\right|\right),\left|\varphi_{x}\left(x, y_{1}\right)-\varphi_{x}\left(x, y_{2}\right)\right| \leqq 6 L\left|y_{1}-y_{2}\right|, \\
\left|\varphi_{y}\left(x_{1}, y\right)-\varphi_{y}\left(x_{2}, y\right)\right| \leqq 6 L\left|y_{1}-y_{2}\right|,\left|\varphi_{y}\left(x, y_{1}\right)-\varphi_{y}\left(x, y_{2}\right)\right| \leqq \eta_{1}\left(\left|y_{1}-y_{2}\right|\right) .
\end{gathered}
$$

Then the vector functions $\varphi \in K$ satisfy relations (20), and then $|\varphi| \leqq M_{1},\left|\varphi_{x}\right| \leqq M_{2}$, $\left|\varphi_{y}\right| \leqq M_{3}$ everywhere in $A$. As a consequence the vector function

$$
F(x, y)=f\left(x, y, \varphi(x, y), \varphi_{x}(x, y), \varphi_{y}(x, y)\right),(x, y) \in A,
$$

is defined everywhere in $A$ and is continuous in $A$.

For $\varphi \in K$ the vector functions $m(y)$ and $n(x)$ defined by (17) and (18) are continuous in $[0, T]$. With $\mu$ defined by (16), the vector function

$$
\psi(x, y)=u_{0}(x)+v_{0}(y)+\int_{0}^{x} \int_{0}^{y}[F(\xi, \eta)-m(\eta)-n(\check{\zeta})-\mu] d \xi d \eta
$$

is continuous in $A$ together with its partial derivatives

$$
\begin{aligned}
& \psi_{x}(x, y)=u_{0}^{\prime}(x)+\int_{0}^{y}[F(x, \eta)-m(\eta)-n(x)-\mu] d \eta \\
& \psi_{y}(x, y)=v_{0}^{\prime}(y)+\int_{0}^{x}[F(\xi, y)-m(y)-n(\xi)-\mu] d \xi
\end{aligned}
$$


Thus, relations (16), (17), (18), (26), and (27) define a map $\mathscr{T}: \psi=\mathscr{T} \varphi$, or $\mathscr{T}: K \rightarrow E$. Let us prove that actually $\mathscr{T}: K \rightarrow K$.

Since $|f| \leqq L$, by (16), (17), (18) we deduce

$$
|\mu| \leqq L, \quad|m(y)|,|n(x)| \leqq 2 L .
$$

On the other hand, by (6), (8), (19), (25), (26), (27), (28), (29), we have with the usual conventions

$$
\begin{gathered}
F(0, y)=F(T, y), \quad F(x, 0)=F(T, 0), \quad m(0)=m(T), \quad n(0)=n(T), \\
\int_{0}^{T} m(\eta) d \eta=0, \quad \int_{0}^{T} n(\xi) d \xi=0 . \\
\psi(x, 0)=\psi(x, T)=u_{0}(x), \quad \psi_{y}(x, 0)=\psi_{y}(x, T), \\
\psi(0, y)=\psi(T, y)=u_{0}(0)+v_{0}(y), \psi_{x}(0, y)=\psi_{x}(T, y), \\
\left|\psi\left(x_{1}, y_{1}\right)-\psi\left(x_{1}, y_{2}\right)-\psi\left(x_{2}, y_{1}\right)+\psi\left(x_{2}, y_{2}\right)\right| \\
=\left|\int_{x_{1}}^{x_{2}} \int_{y_{1}}^{y_{2}}[F(\xi, \eta)-m(\eta)-n(\xi)-\mu] d \xi d \eta\right| \leqq 6 L\left|x_{1}-x_{2}\right|\left|y_{1}-y_{2}\right|
\end{gathered}
$$

In other words $\psi=\mathscr{T} \varphi$ for $\varphi \in K$ satisfies relations (19) and, hence, relations (20) as proved above. Also, we have

and hence

$$
\begin{aligned}
\left|\psi_{x}\left(x, y_{1}\right)-\psi_{x}\left(x, y_{2}\right)\right| & =\left|\int_{y_{1}}^{y_{2}}[F(x, \eta)-m(\eta)-n(x)-\mu] d \eta\right|, \\
\left|\psi_{y}\left(x_{1}, y\right)-\psi_{y}\left(x_{2}, y\right)\right| & =\left|\int_{x_{1}}^{x_{2}}[F(\xi, y)-m(y)-n(\xi)-\mu] d \xi\right|,
\end{aligned}
$$

$\left|\psi_{x}\left(x, y_{1}\right)-\psi_{x}\left(x, y_{2}\right)\right| \leqq 6 L\left|y_{1}-y_{2}\right|, \quad\left|\psi_{y}\left(x_{1}, y\right)-\psi_{y}\left(x_{2}, y\right)\right| \leqq 6 L\left|x_{1}-x_{2}\right|$.

Further, from (17) we have

$$
\begin{aligned}
\left|m\left(y_{1}\right)-m\left(y_{2}\right)\right|= & \mid T^{-1} \int_{0}^{T}\left[f\left(\xi, y_{1}, \varphi\left(\xi, y_{1}\right), \varphi_{x}\left(\xi, y_{1}\right), \varphi_{y}\left(\xi, y_{1}\right)\right)\right. \\
& \left.-f\left(\xi, y_{2}, \varphi\left(\xi, y_{2}\right), \varphi_{x}\left(\xi, y_{2}\right), \varphi_{y}\left(\xi, y_{2}\right)\right)\right] d \xi \mid \\
\leqq & T^{-1} \int_{0}^{T}\left[\omega_{2}\left(\left|y_{1}-y_{2}\right|\right)+\omega_{3}\left(\left|\varphi\left(\xi, y_{1}\right)-\varphi\left(\xi, y_{2}\right)\right|\right)+\right. \\
& \left.+b_{1}\left|\varphi_{x}\left(\xi, y_{1}\right)-\varphi_{x}\left(\xi, y_{2}\right)\right|+b_{2}\left|\varphi_{y}\left(\xi, y_{1}\right)-\varphi_{y}\left(\xi, y_{2}\right)\right|\right] d \xi \\
\leqq & \omega_{2}\left(\left|y_{1}-y_{2}\right|\right)+\omega_{3}\left(M_{3}\left|y_{1}-y_{2}\right|\right)+6 L b_{1}\left|y_{1}-y_{2}\right|+ \\
& +b_{2} \eta_{1}\left(\left|y_{1}-y_{2}\right|\right),
\end{aligned}
$$


and analogously

$\left|n\left(x_{1}\right)-n\left(x_{2}\right)\right| \leqq \omega_{1}\left(\left|x_{1}-x_{2}\right|\right)+\omega_{3}\left(M_{2}\left|x_{1}-x_{2}\right|\right)+b_{1} \eta_{2}\left(\left|x_{1}-x_{2}\right|\right)+6 L b_{2}\left|x_{1}-x_{2}\right|$.

We have now from (29) and (23)

$$
\begin{aligned}
\left|\psi_{y}\left(x, y_{1}\right)-\psi_{y}\left(x, y_{2}\right)\right|= & \mid v_{0}^{\prime}\left(y_{1}\right)-v_{0}^{\prime}\left(y_{2}\right)+ \\
& +\int_{0}^{x}\left[f\left(\xi, y_{1}, \varphi\left(\xi, y_{1}\right), \varphi_{x}\left(\xi, y_{1}\right), \varphi_{y}\left(\xi, y_{1}\right)\right)-m\left(y_{1}\right)-\right. \\
& \left.-f\left(\xi, y_{2}, \varphi\left(\xi, y_{2}\right), \varphi_{x}\left(\xi, y_{2}\right), \varphi_{y}\left(\xi, y_{2}\right)\right)+m\left(y_{2}\right)\right] d \xi \mid \\
\leqq & \omega_{5}\left(\left|y_{1}-y_{2}\right|\right)+\int_{0}^{T}\left[\omega_{2}\left(\left|y_{1}-y_{2}\right|\right)+\right. \\
& +\omega_{3}\left(\left|\varphi\left(\xi, y_{1}\right)-\varphi\left(\xi, y_{2}\right)\right|\right)+b_{1}\left|\varphi_{x}\left(\xi, y_{1}\right)-\varphi_{x}\left(\xi, y_{2}\right)\right|+ \\
& \left.+b_{2}\left|\varphi_{y}\left(\xi, y_{1}\right)-\varphi_{y}\left(\xi, y_{2}\right)\right|+\left|m\left(y_{1}\right)-m\left(y_{2}\right)\right|\right] d \xi \\
\leqq & \omega_{5}\left(\left|y_{1}-y_{2}\right|\right)+2 T \omega_{2}\left(\left|y_{1}-y_{2}\right|\right)+2 T \omega_{3}\left(M_{3}\left|y_{1}-y_{2}\right|\right)+ \\
& +12 L T b_{1}\left|y_{1}-y_{2}\right|+2 T b_{2} \eta_{1}\left(\left|y_{1}-y_{2}\right|\right) \\
= & \left(1-2 T b_{2}\right) \eta_{1}\left(\left|y_{1}-y_{2}\right|\right)+2 T b_{2} \eta_{1}\left(\left|y_{1}-y_{2}\right|\right) \\
= & \eta_{1}\left(\left|y_{1}-y_{2}\right|\right) .
\end{aligned}
$$

Analogously, we have

$$
\left|\psi_{x}\left(x_{1}, y\right)-\psi_{x}\left(x_{2}, y\right)\right| \leqq \eta_{2}\left(\left|x_{1}-x_{2}\right|\right) .
$$

Relations (31), (32), (33), (34) show that $\psi=\mathscr{T} \varphi$ for $\varphi \in K$ satisfies all relations (19) and (25). Thus $\psi \in K$, and $\mathscr{T}: K \rightarrow K$.

The transformation $\mathscr{T}: K \rightarrow K, K \subset E$, is continuous in $K$ with respect to the norm $\|\varphi\|$ of $E$. Indeed, for two vector functions $\varphi_{j} \in K, j=1,2$, we have $\psi_{j}=\mathscr{T} \varphi_{j}$, $F_{j}=F_{\varphi_{j}}, m_{j}=m_{\varphi_{j}}(y), n_{j}=n_{\varphi_{j}}(x), \mu_{j}=\mu_{\varphi_{j}}, j=1,2$, and from (16)

$$
\begin{aligned}
\left|\mu_{1}-\mu_{2}\right|=\mid & \mid T^{-2} \int_{0}^{T} \int_{0}^{T}\left[f\left(\xi, \eta, \varphi_{1}(\xi, \eta), \varphi_{1 x}(\xi, \eta), \varphi_{1 y}(\xi, \eta)\right)-\right. \\
& \left.-f\left(\xi, \eta, \varphi_{2}(\xi, \eta), \varphi_{2 x}(\xi, \eta), \varphi_{2 y}(\xi, \eta)\right)\right] d \xi d \eta \mid \\
\leqq & {\left[\omega_{3}\left(\left\|\varphi_{1}-\varphi_{2}\right\|\right)+b_{1}\left\|\varphi_{1}-\varphi_{2}\right\|+b_{2}\left\|\varphi_{1}-\varphi_{2}\right\|\right] . }
\end{aligned}
$$

Then from (17) we have

$$
\begin{aligned}
\left|m_{1}(y)-m_{2}(y)\right|= & \mid T^{-1} \int_{0}^{T}\left[f\left(x, y, \varphi_{1}(x, y), \varphi_{1 x}(x, y), \varphi_{1 y}(x, y)\right)-\right. \\
& \left.-f\left(x, y, \varphi_{2}(x, y), \varphi_{2 x}(x, y), \varphi_{2 y}(x, y)\right)\right] d x-\mu_{1}+\mu_{2} \mid \\
\leqq & 2\left[\omega_{3}\left(\left\|\varphi_{1}-\varphi_{2}\right\|\right)+b_{1}\left\|\varphi_{1}-\varphi_{2}\right\|+b_{2}\left\|\varphi_{1}-\varphi_{2}\right\|\right],
\end{aligned}
$$

and analogously from (18)

$$
\left|n_{1}(x)-n_{2}(x)\right| \leqq 2\left[\omega_{3}\left(\left\|\varphi_{1}-\varphi_{2}\right\|\right)+b_{1}\left\|\varphi_{1}-\varphi_{2}\right\|+b_{2}\left\|\varphi_{1}-\varphi_{2}\right\|\right]
$$


From (27) we deduce

$$
\begin{aligned}
& \left|\psi_{1}(x, y)-\psi_{2}(x, y)\right| \\
& \quad=\left|\int_{0}^{x} \int_{0}^{y}\left[F_{1}(\xi, \eta)-m_{1}(\eta)-n_{1}(\xi)-\mu_{1}-F_{2}(\xi, \eta)+m_{2}(\eta)+n_{2}(\xi)+\mu_{2}\right] d \xi d \eta\right| \\
& \quad \leqq 6 T^{2}\left[\omega_{3}\left(\left\|\varphi_{1}-\varphi_{2}\right\|\right)+\left(b_{1}+b_{2}\right)\left\|\varphi_{1}-\varphi_{2}\right\|\right] .
\end{aligned}
$$

Analogously, from (28) and (29),

$$
\begin{gathered}
\left|\psi_{1 x}(x, y)-\psi_{2 x}(x, y)\right| \leqq 6 T\left[\omega_{3}\left(\left\|\varphi_{1}-\varphi_{2}\right\|\right)+\left(b_{1}+b_{2}\right)\left\|\varphi_{1}-\varphi_{2}\right\|\right], \\
\left|\psi_{1 y}(x, y)-\psi_{2 y}(x, y)\right| \leqq 6 T\left[\omega_{3}\left(\left\|\varphi_{1}-\varphi_{2}\right\|\right)+\left(b_{1}+b_{2}\right)\left\|\varphi_{1}-\varphi_{2}\right\|\right] .
\end{gathered}
$$

Thus $\left\|\psi_{1}-\psi_{2}\right\| \rightarrow 0$ as $\left\|\varphi_{1}-\varphi_{2}\right\| \rightarrow 0$ uniformly in $K$. Finally, the set $K$ is obviously convex, closed and compact with respect to the norm $\|\varphi\|$ of $E$. By SCHAUDER's fixed point theorem we conclude that there is at least an element $\varphi(x, y) \in K$ such that $\varphi=\mathscr{T} \varphi$, or

$$
\begin{aligned}
\varphi(x, y)= & u_{0}(x)+v_{0}(y)+ \\
& +\int_{0}^{x} \int_{0}^{y}\left[f\left(\xi, \eta, \varphi(\xi, \eta), \varphi_{x}(\xi, \eta), \varphi_{y}(\xi, \eta)\right)-m(\eta)-n(\xi)-\mu\right] d \xi d \eta, \\
m(y)= & T^{-1} \int_{0}^{T} f\left(\xi, y, \varphi(\xi, y), \varphi_{x}(\xi, y), \varphi_{y}(\xi, y)\right) d \xi-\mu, \\
n(x)= & T^{-1} \int_{0}^{T} f\left(x, \eta, \varphi(x, \eta), \varphi_{x}(x, \eta), \varphi_{y}(x, \eta)\right) d \eta-\mu, \\
\mu= & T^{-2} \int_{0}^{T} \int_{0}^{T} f\left(\xi, \eta, \varphi(\xi, \eta), \varphi_{x}(\xi, \eta), \varphi_{y}(\xi, \eta)\right) d \xi d \eta,
\end{aligned}
$$

for all $0 \leqq x, y \leqq T$. Obviously $\varphi_{x}, \varphi_{y}, \varphi_{x y}$ exist everywhere in $A$, are continuous in $A$, and, everywhere in $A$, we have

$$
\varphi_{x y}=f\left(x, y, \varphi, \varphi_{x}, \varphi_{y}\right)-m(y)-n(x)-\mu .
$$

Theorem $I$ is thereby proved.

4. Remark 1. If $f$ is Lipschitzian with respect to all variables $x, y, z, p, q$ in $R$, and if $u_{0}^{\prime}(x), v_{0}^{\prime}(y)$ also are Lipschitzian, then $m(y), n(x)$, as well as $\varphi, \varphi_{x}, \varphi_{y}, \varphi_{x y}$ are Lipschitzian. Indeed, if $\omega_{1}(\alpha)=k_{1} \alpha, \omega_{2}(\beta)=k_{2} \beta, \omega_{3}(\gamma)=b_{0} \gamma, \omega_{4}(\alpha)=k_{4} \alpha$, $\omega_{5}(\beta)=k_{5} \beta$, then

$$
\begin{aligned}
& \eta_{1}(\beta)=\left(1-2 T b_{2}\right)^{-1}\left(k_{5}+2 T k_{2}+2 T b_{0} M_{3}+12 L T b_{1}\right) \beta=k_{6} \beta, \\
& \eta_{2}(\alpha)=\left(1-2 T b_{1}\right)^{-1}\left(k_{4}+2 T k_{1}+2 T b_{0} M_{2}+12 L T b_{2}\right) \alpha=k_{7} \alpha,
\end{aligned}
$$

and then

$$
\begin{gathered}
\left|m\left(y_{1}\right)-m\left(y_{2}\right)\right| \leqq\left(k_{2}+b_{0} M_{3}+6 L b_{1}+b_{2} k_{6}\right)\left|y_{1}-y_{2}\right|=k_{8}\left|y_{1}-y_{2}\right|, \\
\left|n\left(x_{1}\right)-n\left(x_{2}\right)\right| \leqq\left(k_{1}+b_{0} M_{2}+b_{1} k_{7}+6 L b_{2}\right)\left|x_{1}-x_{2}\right|=k_{9}\left|x_{1}-x_{2}\right| .
\end{gathered}
$$

Formulas (33), (34), (35) show that $\varphi_{x}, \varphi_{y}$ are also uniformly Lipschitzian and so is $\varphi_{x y}=f-m-n-\mu$. 
5. Remark 2. The conditions of Theorem I do not assure uniqueness, as the following example shows. Take $T=1, u_{0}(x)=0, v_{0}(y)=0, f=|z|^{\frac{1}{2}} \sin 2 \pi x \sin 2 \pi y$, for $0 \leqq x, y \leqq 1$, and all $y, z, p, q$. Then the equation

$$
u_{x y}=|u|^{\frac{1}{2}} \sin 2 \pi x \sin 2 \pi y,
$$

besides the trivial solution $\varphi_{1}(x, y)=0$, has also the solution $\varphi_{2}(x, y)=\left(16 \pi^{4}\right)^{-1} \times$ $\sin ^{4} \pi x \sin ^{4} \pi y, 0 \leqq x, y \leqq 1$, and both satisfy the boundary conditions. Here we have $m_{1}(y)=m_{2}(y)=0, n_{1}(x)=n_{2}(x)=0$. Note that we may take $N=N_{1}=N_{2}=0$, $L=1, M_{1}=1, M_{2}=M_{3}=2, b_{1}=b_{2}=0$. All conditions of Theorem I are satisfied.

6. The Lipschitzian Case. We shall assume now that $\omega_{3}(\gamma)=b_{0}|\gamma|$, so that $f$ is now Lipschitzian in $z, p, q$ with constants $b_{0}, b_{1}, b_{2}$. In this situation, for given boundary values $u_{0}(x), v_{0}(x)$ and different functions $\varphi_{1}, \varphi_{2} \in K$ we have

$$
\begin{gathered}
\left|\mu_{1}-\mu_{2}\right| \leqq\left(b_{0}+b_{1}+b_{2}\right)\left\|\varphi_{1}-\varphi_{2}\right\|, \\
\left|m_{1}(y)-m_{2}(y)\right|, \quad\left|n_{1}(x)-n_{2}(x)\right| \leqq 2\left(b_{0}+b_{1}+b_{2}\right)\left\|\varphi_{1}-\varphi_{2}\right\|, \\
\left|\psi_{1}(x, y)-\psi_{2}(x, y)\right| \leqq 6 T^{2}\left(b_{0}+b_{1}+b_{2}\right)\left\|\varphi_{1}-\varphi_{2}\right\|, \\
\left|\psi_{1 x}(x, y)-\psi_{2 x}(x, y)\right|, \quad\left|\psi_{1 y}(x, y)-\psi_{2 y}(x, y)\right| \leqq 6 T\left(b_{0}+b_{1}+b_{2}\right)\left\|\varphi_{1}-\varphi_{2}\right\| .
\end{gathered}
$$

Thus

$$
\left\|\psi_{1}-\psi_{2}\right\|=\left\|\mathscr{T} \varphi_{1}-\mathscr{T} \varphi_{2}\right\| \leqq 6 T(T+2)\left(b_{0}+b_{1}+b_{2}\right)\left\|\varphi_{1}-\varphi_{2}\right\| .
$$

If we assume now that

$$
6 T(T+2)\left(b_{0}+b_{1}+b_{2}\right)<1,
$$

then $\mathscr{T}: K \rightarrow K$ is a contraction into. This remark yields

7. Theorem II (uniqueness). Under the same hypotheses of Theorem I, if $\omega_{3}(\gamma)=b_{0}|\gamma|$, and (37) holds, then $\mathscr{T}: K \rightarrow K$ is a contraction, and problem (11)-(18) has one and only one solution.

The boundary values are represented by the pair of functions $w=\left(u_{0}(x), v_{0}(y)\right)$ of class $C^{1}$ and satisfying (6) and (7). Therefore, they form a subset $\mathscr{B}$ of the linear space of all $w$ of class $C^{1}$ satisfying (6) only, and we take in this linear space the norm

$$
\|w\|=\max \left|u_{0}(x)\right|+\max \left|u_{0}^{\prime}(x)\right|+\max \left|v_{0}(y)\right|+\max \left|v_{0}^{\prime}(y)\right| .
$$

The solution of the problem (11)-(18) is actually the system $W=[\varphi(x, y), m(y)$, $n(x), \mu]$. These quadruples also can be thought of as imbedded in a linear space on which we take the norm

$$
\|W\|=\max |\varphi|+\max \left|\varphi_{x}\right|+\max \left|\varphi_{y}\right|+\max |m|+\max |n|+|\mu| .
$$

We shall prove that the solution, or system $W$, is a continuous function $\mathscr{F}$ of the boundary values, or system $w$, and we write

We shall need the numbers

$$
W=\mathscr{F} w, \quad w \in \mathscr{B} .
$$$$
\Delta=\left(1-6 T b_{1}\right)\left(1-6 T b_{2}\right)-36 T^{2} b_{1} b_{2}, \quad k=6 T^{2} b_{0}+72 T^{3} \Delta^{-1} b_{0}\left(b_{1}+b_{2}\right) .
$$ 
8. Theorem III (continuous dependence upon the boundary values). Under the conditions of Theorem II, if in addition $\Delta>0$, and $0<k<1$, then the solution $W=$ $(\varphi, m, n, \mu)$ of problem (11)-(18) is a continuous function $\mathscr{F}$ of the boundary values $w=\left(u_{0}, v_{0}\right) \in \mathscr{B}$ in the topologies determined by the norms (38) and (39).

9. Proof of Theorem III. Let $w_{1}=\left[u_{01}(x), v_{01}(y)\right], w_{2}=\left[u_{02}(x), v_{02}(y)\right]$ be a pair of boundary values as in Theorems I and II, and let $W_{1}=\left[\varphi_{1}, m_{1}, n_{1}, \mu_{1}\right]$, $W_{2}=\left[\varphi_{2}, m_{2}, n_{2}, \mu_{2}\right]$ be the corresponding solutions. Let

$$
\begin{aligned}
\varepsilon= & \left\|w_{1}-w_{2}\right\|=\max \left|u_{01}(x)-u_{02}(x)\right|+\max \left|u_{01}^{\prime}(x)-u_{02}^{\prime}(x)\right|+ \\
& \quad+\max \left|v_{01}(y)-v_{02}(y)\right|+\max \left|v_{01}^{\prime}(y)-v_{02}^{\prime}(y)\right|, \\
\alpha= & \max \left|\varphi_{1}(x, y)-\varphi_{2}(x, y)\right|, \quad \beta=\max \left|\varphi_{1 x}(x, y)-\varphi_{2 x}(x, y)\right|, \\
\gamma= & \max \left|\varphi_{1 y}(x, y)-\varphi_{2 y}(x, y)\right|, \quad \delta=\max \left|m_{1}(y)-m_{2}(y)\right|, \\
\delta^{\prime}= & \max \left|n_{1}(x)-n_{2}(x)\right|, \quad \delta^{\prime \prime}=\left|\mu_{1}-\mu_{2}\right| .
\end{aligned}
$$

We shall denote by $F_{1}$ and $F_{2}$ the functions $F$ relative to $\varphi_{1}$ and $\varphi_{2}$. Then we have

$$
\begin{array}{r}
\delta^{\prime \prime}=\left|\mu_{1}-\mu_{2}\right|=\left|T^{-2} \int_{0}^{T} \int_{0}^{T}\left[F_{1}(x, y)-F_{2}(x, y)\right] d x d y\right| \leqq b_{0} \alpha+b_{1} \beta+b_{2} \gamma, \\
\left|m_{1}(y)-m_{2}(y)\right|=\left|T^{-1} \int_{0}^{T}\left[F_{1}(x, y)-F_{2}(x, y)\right] d x-\mu_{1}+\mu_{2}\right| \leqq b_{0} \alpha+b_{1} \beta+b_{2} \gamma+\delta^{\prime \prime}, \\
\left|n_{1}(x)-n_{2}(x)\right|=\left|T^{-1} \int_{0}^{T}\left[F_{1}(x, y)-F_{2}(x, y)\right] d y-\mu_{1}+\mu_{2}\right| \leqq b_{0} \alpha+b_{1} \beta+b_{2} \gamma+\delta^{\prime \prime} .
\end{array}
$$

Hence

Analogously,

$$
\delta^{\prime \prime} \leqq b_{0} \alpha+b_{1} \beta+b_{2} \gamma, \quad \delta, \delta^{\prime} \leqq b_{0} \alpha+b_{1} \beta+b_{2} \gamma+\delta^{\prime \prime} \text {. }
$$

$$
\begin{aligned}
&\left|\varphi_{1}(x, y)-\varphi_{2}(x, y)\right|=\mid u_{01}(x)+v_{01}(y)-u_{02}(x)-v_{02}(y)+ \\
&+\int_{0}^{x} \int_{0}^{y}\left[F_{1}(x, y)-m_{1}(y)-n_{1}(x)-\mu_{1}-F_{2}(x, y)+m_{2}(y)+n_{2}(x)+\mu_{2}\right] d x d y \mid \leqq \\
& \quad \varepsilon+T^{2}\left(b_{0} \alpha+b_{1} \beta+b_{2} \gamma+\delta+\delta^{\prime}+\delta^{\prime \prime}\right) \\
&\left|\varphi_{1 x}(x, y)-\varphi_{2 x}(x, y)\right| \leqq \varepsilon+T\left(b_{0} \alpha+b_{1} \beta+b_{2} \gamma+\delta+\delta^{\prime}+\delta^{\prime \prime}\right) \\
&\left|\varphi_{1 y}(x, y)-\varphi_{2 y}(x, y)\right| \leqq \varepsilon+T\left(b_{0} \alpha+b_{1} \beta+b_{2} \gamma+\delta+\delta^{\prime}+\delta^{\prime \prime}\right)
\end{aligned}
$$

and hence

$$
\begin{gathered}
\alpha \leqq \varepsilon+T^{2}\left(b_{0} \alpha+b_{1} \beta+b_{2} \gamma+\delta+\delta^{\prime}+\delta^{\prime \prime}\right), \\
\beta, \gamma \leqq \varepsilon+T\left(b_{0} \alpha+b_{1} \beta+b_{2} \gamma+\delta+\delta^{\prime}+\delta^{\prime \prime}\right) .
\end{gathered}
$$

Relations (41), (42) yield

$$
\begin{aligned}
\delta^{\prime \prime} \leqq b_{0} \alpha+b_{1} \beta+b_{2} \gamma, & \delta, \delta^{\prime} \leqq 2\left(b_{0} \alpha+b_{1} \beta+b_{2} \gamma\right), \\
\alpha \leqq \varepsilon+6 T^{2}\left(b_{0} \alpha+b_{1} \beta+b_{2} \gamma\right), & \beta, \gamma \leqq \varepsilon+6 T\left(b_{0} \alpha+b_{1} \beta+b_{2} \gamma\right) .
\end{aligned}
$$


The last relation can be written in the form

$$
\begin{aligned}
& \beta=6 T b_{0} \xi_{1} \alpha+6 T b_{1} \xi_{1} \beta+6 T b_{2} \xi_{1} \gamma+\varepsilon \xi_{1}, \\
& \gamma=6 T b_{0} \xi_{2} \alpha+6 T b_{1} \xi_{2} \beta+6 T b_{2} \xi_{2} \gamma+\varepsilon \xi_{2},
\end{aligned}
$$

where $0 \leqq \xi_{1}, \xi_{2} \leqq 1$ are convenient numbers, and then

$$
\begin{aligned}
\left(1-6 T b_{1} \xi_{1}\right) \beta-6 T b_{2} \xi_{1} \gamma & =6 T b_{0} \xi_{1} \alpha+\varepsilon \xi_{1}, \\
-6 T b_{1} \xi_{2} \beta+\left(1-6 T b_{2} \xi_{2}\right) \gamma & =6 T b_{0} \xi_{2} \alpha+\varepsilon \xi_{2} .
\end{aligned}
$$

If $\Delta^{\prime}$ is the determinant of this system, we have $\Delta^{\prime} \geqq \Delta>0,0<6 T b_{j}<1, j=1,2$, and

$$
\begin{aligned}
\beta & =\Delta^{\prime-1}\left\{\left(1-6 T b_{2} \xi_{2}\right)\left(6 T b_{0} \xi_{1} \alpha+\varepsilon \xi_{1}\right)+\left(6 T b_{2} \xi_{1}\right)\left(6 T b_{0} \xi_{2} \alpha+\varepsilon \xi_{2}\right)\right\} \\
& \leqq 2 \Delta^{-1}\left(\varepsilon+6 T b_{0} \alpha\right) .
\end{aligned}
$$

Analogously, we have

Finally, by (43)

$$
\gamma \leqq 2 \Delta^{-1}\left(\varepsilon+6 T b_{0} \alpha\right)
$$

$$
\alpha \leqq \varepsilon+72 T^{3} \Delta^{-1} b_{0}\left(b_{1}+b_{2}\right) \alpha+6 T^{2} b_{0} \alpha+12 T^{2} \Delta^{-1}\left(b_{1}+b_{2}\right) \varepsilon .
$$

Since the number $k$ defined in (40) lies in the interval $0<k<1$, we have

$$
\alpha \leqq(1-k)^{-1}\left[1+12 \Delta^{-1} T^{2}\left(b_{1}+b_{2}\right)\right] \varepsilon .
$$

This proves that $\alpha, \beta, \gamma, \delta, \delta^{\prime}, \delta^{\prime \prime} \rightarrow 0$ as $\varepsilon \rightarrow 0$ uniformly in $K$. Theorem III is thereby proved.

10. A Method of Successive Approximations. Under the hypotheses of Theorem III, the usual method of successive approximations defined by $\varphi_{k+1}=\mathscr{T} \varphi_{k}$, $k=0,1 \ldots$, converges toward the solution $\varphi$ of problem $(11)-(18)$, where $\varphi_{0}$ is an arbitrary element of $K$. It may be convenient to use as first approximation

Then

$$
\varphi_{0}(x y)=u_{0}(x)+v_{0}(y)
$$

$$
\begin{aligned}
& F_{0}(x, y)=f\left(x, y, u_{0}(x)+v_{0}(y), \quad u_{0}^{\prime}(x), v_{0}^{\prime}(y)\right), \quad \mu_{0}=T^{-2} \int_{0}^{T} \int_{0}^{T} F_{0}(\xi, \eta) d \xi d \eta, \\
& m_{0}(y)=T^{-1} \int_{0}^{T} F_{0}(\xi, y) d \xi, \quad u_{0}(x)=T^{-1} \int_{0}^{T} F_{0}(x, \eta) d \eta, \\
& \varphi_{1}(x, y)=u_{0}(x)+v_{0}(y)+\int_{0}^{x} \int_{0}^{y}\left[F_{0}(\xi, \eta)-m_{0}(\eta)-n_{0}(\xi)-\mu_{0}\right] d \xi d \eta, \\
& \text { and successively, }
\end{aligned}
$$

$$
\begin{aligned}
F_{k}(x, y)= & f\left(x, y, \varphi_{k}(x, y), \varphi_{k x}(x, y)+\varphi_{k y}(x, y)\right), \\
\mu_{k}= & T^{-2} \int_{0}^{T} \int_{0}^{T} F_{k}(\xi, \eta) d \xi d \eta, \quad m_{k}(y)=T^{-1} \int_{0}^{T} F_{k}(\xi, y) d \xi \\
n_{k}(x)= & T^{-1} \int_{0}^{T} F_{k}(x, \eta) d \eta, \quad \varphi_{k+1}(x, y)=u_{0}(x)+v_{0}(y)+ \\
& +\int_{0}^{T} \int_{0}^{T}\left[F_{k}(\xi, \eta)-m_{k}(\eta)-n_{k}(\xi)-\mu_{k}\right] d \xi d \eta, \quad k=1,2, \ldots
\end{aligned}
$$


Then we have $\varphi_{k} \rightarrow \varphi, \varphi_{k x} \rightarrow \varphi_{x}, \varphi_{k y} \rightarrow \varphi_{y}, m_{k} \rightarrow m, n_{k} \rightarrow n, \mu_{k} \rightarrow \mu$ uniformly for $0 \leqq x \leqq T, 0 \leqq y \leqq T$, and consequently we have also $\varphi_{k x y} \rightarrow \varphi_{x y}$ as $k \rightarrow \infty$ uniformly.

11. Smoothness of the Solution. Two theorems can now be stated concerning the smoothness of the solution $(\varphi, m, n, \mu)$ of the problem $(11)-(18)$.

( $\alpha$ ) Under the conditions of Theorems I, II, III, if $f(x, y, z, p, q)$ is of class $C^{1}$ in $R$ and $u_{0}(x), v_{0}(y)$ of class $C^{2}$, then $m(y), n(x)$ are of class $C^{1}$ and $\varphi(x, y)$ of class $C^{2}$.

This statement was essentially proved in Section 12 of [2a]. A more precise statement is as follows:

( $\beta$ ) Under the conditions of Theorems I, II, III, if $f(x, y, z, p, q)$ is of class $C^{1}$ with Lipschitzian first order partial derivatives, if $u_{0}(x), v_{0}(y)$ are of class $C^{2}$ with Lipschitzian second order derivatives, then $m(y), n(x)$ are of class $C^{1}$ with Lipschitzian first derivatives and $\varphi(x, y)$ of class $C^{2}$ also with Lipschitzian second order partial derivatives.

The proof is the same as for $(\alpha)$. An analogous statement holds:

$(\gamma)$ Under the conditions of Theorem I, II, III, if $f(x, y, z, p, q)$ is of class $C^{1+r}$ in $R$ with Lipschitzian partial derivatives of the order $1+r$, if $u_{0}(x), v_{0}(y)$ are of class $C^{2+r}$ with Lipschitzian derivatives of the order $2+r$, then $m(y), n(x)$ are of class $C^{1+r}$ with Lipschitzian derivatives of order $1+r$, and $\varphi(x, y)$ is of class $C^{2+r}$ with Lipschitzian partial derivatives of the order $2+r$.

\section{Criteria for the Existence of Periodic Solutions in the Large of the Original Problem}

12. A Differential Equation Containing a Small Parameter. Let us consider the differential equation

$$
\begin{aligned}
u_{x y} & =f\left(x, y, u, u_{x}, u_{y}\right), \\
f & =\varepsilon\left[\psi(x, y)+C u+\psi_{1}(y) u_{x}+\psi_{2}(x) u_{y}\right]+\varepsilon^{2} g\left(x, y, u, u_{x}, u_{y}\right),
\end{aligned}
$$

where $\varepsilon$ is a small parameter, and $\psi, \psi_{1}, \psi_{2}, g$ are periodic functions of period $T=2 \pi / \omega$ in $x$ and $y$. The Fourier series of $\psi, \psi_{1}, \psi_{2}$ will be denoted by

$$
\begin{aligned}
\psi(x, y) & \sim \sum_{m, n=0}^{\infty}\left(a_{m n} \cos m \omega x \cos n \omega y+b_{m n} \cos m \omega x \sin n \omega y+\right. \\
& \left.+c_{m n} \sin m \omega x \cos n \omega y+d_{m n} \sin m \omega x \sin n \omega y\right), \\
\psi_{1}(y) & \sim e_{0}+\sum_{1}^{\infty}\left(e_{n} \cos n \omega y+f_{n} \sin n \omega y\right), \\
\psi_{2}(x) & \sim g_{0}+\sum_{1}^{\infty}\left(g_{n} \cos n \omega x+h_{n} \sin n \omega x\right) .
\end{aligned}
$$

If $u_{0}(x), v_{0}(y)$ denote arbitrary boundary values with $v_{0}(0)=0$, and $u_{0}, v_{0}$ both of class $C^{1}$ and $u_{0}^{\prime}, v_{0}^{\prime}$ Lipschitzian with constants $k_{1}, k_{2}$ respectively, then it is 
convenient for us to denote their Fourier series as follows:

$$
\begin{aligned}
& u_{0}(x) \sim \alpha_{0}+\sum_{1}^{\infty}\left(\alpha_{n} \cos n \omega x-\alpha_{n}+\beta_{n} \sin n \omega x\right), \\
& v_{0}(y) \sim \sum_{1}^{\infty}\left(\gamma_{n} \cos n \omega y-\gamma_{n}+\delta_{n} \sin n \omega y\right),
\end{aligned}
$$

where both series $\sum \alpha_{m}, \sum \gamma_{n}$ are absolutely convergent. With this notation we have

$$
\begin{gathered}
u_{0}(0)=\alpha_{0}, \quad v_{0}(0)=0, \quad T^{-1} \int_{0}^{T} u_{0}(x) d x=\alpha_{0}-\sum_{1}^{\infty} \alpha_{n}, \\
T^{-1} \int_{0}^{T} v_{0}(y) d y=-\sum_{1}^{\infty} \gamma_{n} .
\end{gathered}
$$

If we apply formally the method of successive approximations of Section 10 to equation (46) with initial values $u_{0}(x), v_{0}(y)$, we obtain at the first approximation and preserving only the terms in $\varepsilon$, namely a quadruple $\left[\varphi_{0}, \varepsilon m_{0}, \varepsilon n_{0}\right.$, $\left.\varepsilon \mu_{0}\right]$, with $\varphi_{0}, m_{0}, n_{0}, \mu_{0}$ given by

$$
\begin{aligned}
\varphi_{0}(x, y) & =u_{0}(x)+v_{0}(y)+\varepsilon \int_{0}^{x} \int_{0}^{y}\left[F_{0}(\xi, \eta)-m_{0}(\xi)-n_{0}(\eta)-\mu_{0}\right] d \xi d \eta, \\
m_{0}(y) & =T^{-1} \int_{0}^{T} F_{0}(\xi, y) d \xi-\mu_{0}, \quad n_{0}(x)=T^{-1} \int_{0}^{T} F_{0}(x, \eta) d \eta-\mu_{0}, \\
\mu_{0} & =T^{-2} \int_{0}^{T} \int_{0}^{T} F_{0}(\xi, \eta) d \xi d \eta, \\
F_{0}(x, y) & =\psi(x, y)+C u_{0}(x)+C v_{0}(y)+\psi_{1}(y) u_{0}^{\prime}(x)+\psi_{2}(x) v_{0}^{\prime}(y) .
\end{aligned}
$$

If we write

$$
\begin{gathered}
m_{0}(y) \sim \sum_{1}^{\infty}\left(B_{n} \cos n \omega y+C_{n} \sin n \omega y\right), \quad n_{0}(x) \sim \sum_{1}^{\infty}\left(D_{n} \cos n \omega x+E_{n} \sin n \omega x\right), \\
\mu_{0}=A_{0},
\end{gathered}
$$

we obtain

$$
\begin{gathered}
\mu_{0}=A_{0}=a_{00}+C\left(\alpha_{0}-\sum_{1}^{\infty} \alpha_{s}-\sum_{1}^{\infty} \gamma_{s}\right), \\
m_{0}(y)=a_{00}+\kappa_{1}(y)+C\left(\alpha_{0}-\sum_{1}^{\infty} \alpha_{s}\right)+C v_{0}(y)+g_{0} v_{0}^{\prime}(y)-\mu_{0}, \\
n_{0}(x)=a_{00}+\kappa_{2}(x)+C u_{0}(x)+e_{0} u_{0}^{\prime}(x)+C\left(-\sum_{1}^{\infty} \gamma_{s}\right)-\mu_{0}, \\
\kappa_{1}(y)=T^{-1} \int_{0}^{T} \psi(\xi, y) d \xi-a_{00} \sim \sum_{1}^{\infty}\left(a_{0 n} \cos n \omega y+b_{0 n} \sin n \omega y\right), \\
\kappa_{2}(x)=T^{-1} \int_{0}^{T} \psi(x, \eta) d \eta-a_{00} \sim \sum_{1}^{\infty}\left(a_{n_{0}} \cos n \omega x+c_{\pi_{0}} \sin n \omega x\right) .
\end{gathered}
$$


In terms of the Fourier constants relations (49) become

$$
\begin{array}{lll}
A_{0}=a_{00}+C\left(\alpha_{0}-\sum_{1}^{\infty} \alpha_{s}-\sum_{1}^{\infty} \gamma_{s}\right), & B_{n}=C \gamma_{n}+n \omega g_{0} \delta_{n}+a_{0 n}, \\
C_{n}=C \delta_{n}-n \omega g_{0} \gamma_{n}+b_{0 n}, & D_{n}=C \alpha_{n}+n \omega e_{0} \beta_{n}+a_{n 0}, \\
E_{n}=C \beta_{n}-n \omega e_{0} \alpha_{n}+c_{n 0}, & n=1,2, \ldots .
\end{array}
$$

We shall denote by $u(x), v(y)$ the excesses of the functions $u_{0}(x), v_{0}(y)$ over their mean values:

$$
\begin{aligned}
& u(x)=u_{0}(x)-\alpha_{0}+\sum_{1}^{\infty} \alpha_{s} \sim \sum_{1}^{\infty}\left(\alpha_{s} \cos s \omega x+\beta_{s} \sin s \omega x\right), \\
& v(y)=v_{0}(y)+\sum_{1}^{\infty} \gamma_{s} \sim \sum_{1}^{\infty}\left(\gamma_{s} \cos s \omega y+\delta_{s} \sin s \omega y\right) .
\end{aligned}
$$

If we require $\mu_{0}=0, m_{0}(y) \equiv 0, n_{0}(x) \equiv 0$, then relations (49) reduce to

$$
C v(y)+g_{0} v^{\prime}(y)=-\kappa_{1}(y), \quad C u(x)+e_{0} u^{\prime}(x)=-\kappa_{2}(x) .
$$

For $e_{0}=0$, we have

for $e_{0} \neq 0$, we have

$$
u(x)=-C^{-1} \kappa_{2}(x)
$$

$$
\begin{aligned}
u(x)= & \exp \left(-e_{0}^{-1} C x\right)\left[K+e_{0}^{-1} \int_{0}^{x} \exp \left(e_{0}^{-1} C \xi\right) \kappa_{2}(\xi) d \xi\right], \\
K= & -e_{0}^{-2} C\left(1-\exp \left(-e_{0}^{-1} C T\right)\right)^{-1} \int_{0}^{r} \exp \left(e_{0}^{-1} C x\right) d x \times \\
& \times \int_{0}^{x} \exp \left(e_{0}^{-1} C \xi\right) \kappa_{2}(\xi) d \zeta
\end{aligned}
$$

where the constant $K$ is determined in such a way that

$$
\int_{0}^{T} u(x) d x=0
$$

Analogous relations hold for $v(y)$. This determines all the coefficients $\alpha_{n}, \beta_{n}, \gamma_{n}, \delta_{n}$, $n=1,2, \ldots$. Actually, relations (53), (54) are equivalent to those we obtain from (51) by taking $B_{n}=C_{n}=D_{n}=E_{n}=0$ and solving with respect to $\alpha_{n}, \beta_{n}, \gamma_{n}, \delta_{n}$ :

$$
\begin{aligned}
& \alpha_{n}=\left(C^{2}+n^{2} \omega^{2} e_{0}^{2}\right)^{-1}\left(-C a_{n 0}+n \omega e_{0} c_{n 0}\right), \\
& \beta_{n}=\left(C^{2}+n^{2} \omega^{2} e_{0}^{2}\right)^{-1}\left(-n \omega e_{0} a_{n 0}-C c_{n 0}\right), \\
& \gamma_{n}=\left(C^{2}+n^{2} \omega^{2} g_{0}^{2}\right)^{-1}\left(-C a_{0 n}+n \omega g_{0} b_{0 n}\right), \\
& \delta_{n}=\left(C^{2}+n^{2} \omega^{2} g_{0}^{2}\right)^{-1}\left(-n \omega g_{0} a_{0 n}-C b_{0 n}\right), \\
& n=1,2, \ldots
\end{aligned}
$$

The coefficients $\alpha_{n}, \beta_{n}, \gamma_{n}, \delta_{n}, n=1,2, \ldots$, being so determined, then equation $\mu_{0}=A_{0}=0$ yields

$$
\alpha_{0}=-C^{-1} a_{00}+\sum_{1}^{\infty} \alpha_{s}+\sum_{1}^{\infty} \gamma_{s},
$$


provided the series $\sum \alpha_{s}, \sum \gamma_{s}$ converge. This will be the case under the hypotheses of Criterion I below. We shall denote the corresponding functions $u_{0}(x), v_{0}(y)$ so determined by $U(x), V(y)$, or

$$
\begin{aligned}
& U(x)=u(x)+\alpha_{0}-\sum_{1}^{\infty} \alpha_{s} \sim \alpha_{0}+\sum_{1}^{\infty}\left(\alpha_{s} \cos s \omega x-\alpha_{s}+\beta_{s} \sin s \omega x\right), \\
& V(y)=v(y)-\sum_{1}^{\infty} \gamma_{s} \sim \sum_{1}^{\infty}\left(\gamma_{s} \cos s \omega y-\gamma_{s}+\delta_{s} \sin s \omega y\right) .
\end{aligned}
$$

Under the conditions of Criterion I we shall require that these functions be interior points of the set defined by relations (7) of Theorem I.

13. Criterion I. If the function $f$ given in (46) for all $|\varepsilon|<\varepsilon_{0}$ satisfies all conditions of Theorems I, II, III with given constants $T, N, N_{1}, N_{2}, L, M_{1}, M_{2}$, $M_{3}, b_{0}, b_{1}, b_{2}$, and in addition if $f$ is Lipschitzian with respect to $x$ and $y$ in $R$, if $C \neq 0$, and the functions $\kappa_{1}(x), \kappa_{2}(y), U(x), V(y)$ defined in (50) and (55) are of class $C^{1}$ with Lipschitzian first derivatives, and

$$
\begin{gathered}
|U(0)| \leqq N_{0}<N, \quad\left|U\left(x_{1}\right)-U\left(x_{2}\right)\right| \leqq N_{10}\left|x_{1}-x_{2}\right|, \quad N_{10}<N_{1}, \\
\left|V\left(y_{1}\right)-V\left(y_{2}\right) \leqq N_{20}\right| y_{1}-y_{2} \mid, \quad N_{20}<N_{2},
\end{gathered}
$$

then there is some $\bar{\varepsilon}_{0}, 0<\bar{\varepsilon}_{0} \leqq \varepsilon_{0}$, such that equation (46) for all $|\varepsilon| \leqq \bar{\varepsilon}_{0}$ possesses at least one periodic solution $\varphi(x, y)$ of period $T$ in $x$ and $y$, which is Lipschitzian in $E_{2}$ together with $\varphi_{x}, \varphi_{y}, \varphi_{x y}$ :

$$
\varphi_{x y}=f\left(x, y, \varphi, \varphi_{x}, \varphi_{y}\right), \quad \varphi(x+T, y)=\varphi(x, y)=\varphi(x, y+T) .
$$

Moreover, the periodic functions $u_{0}(x)=\varphi(x, 0)=\varphi(x, T), v_{0}(y)=\varphi(0, y)-\varphi(0,0)$ $=\varphi(T, y)-\varphi(T, 0)$, satisfy relations $(7)$ of Theorem $\mathrm{I}$.

14. Proof of Criterion I. Let us denote by $k_{40}, k_{50}$ the Lipschitzian constants of $U(x)$ and $V(y)$ respectively, and let $k_{4}, k_{5}$ be arbitrary numbers $k_{4}>k_{40}$, $k_{5}>k_{50}$. Let us denote as usual by $k_{1}, k_{2}$ the Lipschitzian constants of $f$ with respect to $x$ and $y$ respectively in $R$.

Let $S$ be the set of all pairs $w=\left[u_{0}(x), v_{0}(y)\right]$ of functions $u_{0}(x), v_{0}(y)$ periodic of period $T$, of class $C^{1}$, with derivatives $u_{0}^{\prime}(x), v_{0}^{\prime}(y)$ Lipschitzian of constants $k_{4}, k_{5}$, and satisfying relations (7) of Theorem I, that is

$$
\begin{gathered}
\left|u_{0}(0)\right| \leqq N, \quad\left|u_{0}\left(x_{1}\right)-u_{0}\left(x_{2}\right)\right| \leqq N_{1}\left|x_{1}-x_{2}\right|, \\
v_{0}(0)=0, \quad\left|v_{0}\left(y_{1}\right)-v_{0}\left(y_{2}\right)\right| \leqq N_{2}\left|y_{1}-y_{2}\right| .
\end{gathered}
$$

Then $w_{0}=[U, V] \in S$. We shall consider $S$ imbedded in the Banach space of all pairs of periodic fuctions of class $C^{1}$ with norm

$$
\|w\|=\max |u(x)|+\max \left|u^{\prime}(x)\right|+\max |v(y)|+\max \left|v^{\prime}(y)\right| .
$$

For every $w=\left[u_{0}(x), v_{0}(y)\right] \in S$ we shall determine the solution $W=[\varphi, \varepsilon m, \varepsilon n, \varepsilon \mu]$ of the modified problem relative to (46). Since this solution can be determined by the method of successive approximations of Section 10 , we see that $W$ can be 
written in the form

$$
\begin{aligned}
\kappa(x, y, \varepsilon) & =\varphi_{0}(x, y)+\tilde{\varphi}(x, y, \varepsilon), & m(y, \varepsilon) & =m_{0}(y)+\tilde{m}(y, \varepsilon), \\
n(x, \varepsilon) & =n_{0}(x)+\tilde{n}(x, \varepsilon), & \mu(\varepsilon) & =\mu_{0}+\tilde{\mu}(\varepsilon),
\end{aligned}
$$

where $\tilde{\varphi}, \tilde{m}, \tilde{n}, \tilde{\mu}=O(1)$ uniformly as $\varepsilon \rightarrow 0$ and $\varphi_{0}, m_{0}, n_{0}, \mu_{0}$ are given by formulas (48).

By use of the functions $u(x), v(y)$ as in Section 8 , equations $\mu=0, m(y) \equiv 0$, $n(x) \equiv 0$ reduce to

For $e_{0}=0$ we have

$$
\begin{aligned}
& C v(y)+g_{0} v^{\prime}(y)=-\kappa_{1}(y)-\tilde{m}(y, \varepsilon), \\
& C u(x)+e_{0} u^{\prime}(x)=-\kappa_{2}(x)-\tilde{n}(x, \varepsilon), \\
& a_{00}+C\left(\alpha_{0}-\sum_{1}^{\infty} \alpha_{s}-\sum_{1}^{\infty} \gamma_{s}\right)-\tilde{\mu}(\varepsilon)=0 .
\end{aligned}
$$

for $e_{0} \neq 0$ we have

$$
u(x)=-C^{-1}\left(\kappa_{2}(x)+\tilde{n}(x, \varepsilon)_{n}\right.
$$

$$
\begin{aligned}
u(x)= & \exp \left(-e_{0}^{-1} C x\right)\left[K+e_{0}^{-1} \int_{0}^{x} \exp \left(e_{0}^{-1} C \xi\right)\left(\kappa_{2}(\xi)+\tilde{n}(\xi, \varepsilon)\right) d \xi\right], \\
K= & -e_{0}^{-2} C\left(1-\exp \left(-e_{0}^{-1} C T\right)\right)^{-1} \int_{0}^{T} \exp \left(e_{0}^{-1} C x\right) d x \int_{0}^{x} \exp \left(e_{0}^{-1} C \xi\right) \times \\
& \times\left(\kappa_{2}(\xi)+\tilde{n}(\xi, \varepsilon)\right) d \xi,
\end{aligned}
$$

and hence

$$
\begin{array}{lll}
u^{\prime}(x)=-C^{-1}(d / d x)\left(\kappa_{2}(x)+\tilde{n}(x, \varepsilon)\right) & \text { if } & e_{0}=0, \\
u^{\prime}(x)=-e_{0}^{-1}\left(C u(x)+\kappa_{2}(x)+\tilde{n}(x, \varepsilon)\right) & \text { if } & e_{0} \neq 0 .
\end{array}
$$

Analogous formulas hold for $v(y)$.

This determines $u(x), v(y)$ and hence all coefficients $\alpha_{n}, \beta_{n}, \gamma_{n}, \delta_{n}, n=1,2, \ldots$ By Remark 1 we know that $m, n$ are Lipschitzian functions, and so are $\tilde{m}, \tilde{n}$ as well as $\kappa_{1}, \kappa_{2}$. Thus $u(x), u(y)$ are periodic functions of mean value zero, of class $C^{1}$, with Lipschitzian first derivatives. Thus, the series $\sum \alpha_{n}, \sum \gamma_{n}$ are absolutely convergent, and (47), (57) yield

$$
\begin{aligned}
\alpha_{0} & =-C^{-1}\left(a_{00}-\tilde{\mu}(\varepsilon)\right)+\sum_{1}^{\infty} \alpha_{s}+\sum_{1}^{\infty} \gamma_{s}, \\
& \left.=-C^{-1}\left(a_{00}-\tilde{\mu}(\varepsilon)\right)-u(0)-v(0)\right), \\
u_{0}(x) & =u(x)+\alpha_{0}-\sum_{1}^{\infty} \alpha_{s}, \quad v_{0}(y)=v(y)-\sum_{1}^{\infty} \gamma_{s} .
\end{aligned}
$$

Note that these functions, when we take $\tilde{m}=\tilde{n}=0$, reduce to $U(x)$ and $V(y)$ respectively, and thus the convergence of the series $\sum \alpha_{n}, \sum \gamma_{n}$ of Section 12 is proved above.

Actually, for every $w=[u(x), v(y)] \in S$, we can first determine $m, n, \mu$ as in Theorems I, II, III, using the method of successive approximations of Section 10; then we determine $\tilde{m}, \tilde{n}$, and finally the second members of formulae (58), (59), 
(60), (62), and analogous ones determine new functions, say $\bar{w}=\left[\bar{u}_{0}(x), \bar{v}_{0}(y)\right]$. Thus, we have a map $\mathscr{F}$,

$$
\bar{w}=\mathscr{F}_{w}, \quad w \in S,
$$

whose fixed elements $w=\mathscr{F} w$, if any, have the property that $\mu=0, m(y) \equiv 0$, $n(x) \equiv 0$.

We have already chosen the uniform topology of class $C^{1}$ on $w$ and $\bar{w}$ by means of (56). Let us choose the uniform topology of class $C^{0}$ on $m, n$ as in Theorem III, as well as on $\tilde{m}, \tilde{n}$. We know already from Theorem III that $m, n$ are continuous functions of $w$, and so are $\tilde{m}, \tilde{n}$. The second members of (58), (59), (60), (62) define continuous functions of $\tilde{m}, \tilde{n}$. Thus $\mathscr{F}$ is a continuous function of $w$ for $w \in S$ in the topology defined by (56).

By Theorems I, II, III we know that $m(y, \varepsilon), n(x, \varepsilon)$ are Lipschitzian functions. The same property holds for $\tilde{m}(y, \varepsilon), \tilde{n}(x, \varepsilon)$, but these functions - as well as their Lipschitzian constants - have a uniform bound of the form $M \varepsilon$ for some $M>0$ and all $|\varepsilon|<\varepsilon_{0}$. Then, by choosing convenient constants $k, k_{1}$, we have

$$
\begin{gathered}
|\tilde{m}(y, \varepsilon)|,|\tilde{n}(x, \varepsilon)| \leqq k \varepsilon, \quad|\tilde{\mu}| \leqq k \varepsilon, \quad\left|\tilde{m}\left(y_{1}, \varepsilon\right)-\tilde{m}\left(y_{2}, \varepsilon\right)\right| \leqq k \varepsilon\left|y_{1}-y_{2}\right|, \\
\left|\tilde{n}\left(x_{1}, \varepsilon\right)-\tilde{n}\left(x_{2}, \varepsilon\right)\right| \leqq k \varepsilon\left|x_{1}-x_{2}\right|, \quad\left|\bar{u}_{0}-U\right| \leqq k_{1} \varepsilon, \quad\left|\bar{v}_{0}-V\right| \leqq k_{1} \varepsilon, \\
\left|\bar{u}_{0}^{\prime}-U^{\prime}\right| \leqq k_{1} \varepsilon, \quad\left|\bar{v}_{0}^{\prime}-V^{\prime}\right| \leqq k_{1} \varepsilon, \quad\left|\bar{u}_{0}\left(x_{1}\right)-U\left(x_{1}\right)-\bar{u}_{0}\left(x_{2}\right)+U\left(x_{2}\right)\right| \leqq k_{1} \varepsilon, \\
\left|\bar{v}_{0}\left(y_{1}\right)-V\left(y_{1}\right)-\bar{v}\left(y_{2}\right)+V\left(y_{2}\right)\right| \leqq k_{1} \varepsilon, \quad\left|\bar{u}_{0}^{\prime}\left(x_{1}\right)-U^{\prime}\left(x_{1}\right)-\bar{u}_{0}^{\prime}\left(x_{2}\right)+U^{\prime}\left(x_{2}\right)\right| \leqq k_{1} \varepsilon, \\
\left|\bar{v}_{0}^{\prime}\left(y_{1}\right)-V^{\prime}\left(y_{1}\right)-\bar{v}^{\prime}\left(y_{2}\right)+V^{\prime}\left(y_{2}\right)\right| \leqq k_{1} \varepsilon .
\end{gathered}
$$

If $k_{4}, k_{5}$ are the Lipschitzian constants of $U^{\prime}, V^{\prime}$, and

$$
\bar{\varepsilon}_{0}=\min \left[\varepsilon_{0}, k_{1}^{-1}\left(N-N_{0}\right), k_{1}^{-1}\left(N_{1}-N_{10}\right), k_{1}^{-1}\left(N_{2}-N_{20}\right)\right],
$$

then for $|\varepsilon| \leqq \bar{\varepsilon}_{0}$ we have

$$
\begin{aligned}
& \quad\left|\bar{u}_{0}(0)\right| \leqq|U(0)|+k_{1} \varepsilon \leqq N_{0}+k_{1} \varepsilon \leqq N, \\
& \left|\bar{u}_{0}\left(x_{1}\right)-\bar{u}_{0}\left(x_{2}\right)\right| \leqq\left(N_{10}+k_{1} \varepsilon\right)\left|x_{1}-x_{2}\right| \leqq N_{1}\left|x_{1}-x_{2}\right|, \\
& \left|\bar{u}_{0}^{\prime}\left(x_{1}\right)-\bar{u}_{0}^{\prime}\left(x_{2}\right)\right| \leqq\left(k_{4}+k_{1} \varepsilon\right)\left|x_{1}-x_{2}\right|, \\
& \left|\bar{v}_{0}\left(y_{1}\right)-\bar{v}_{0}\left(y_{2}\right)\right| \leqq\left(N_{20}+k_{1} \varepsilon\right)\left|y_{1}-y_{2}\right| \leqq N_{2}\left|y_{1}-y_{2}\right|, \\
& \left|\bar{v}_{0}^{\prime}\left(y_{1}\right)-\bar{v}_{0}^{\prime}\left(y_{2}\right)\right| \leqq\left(k_{5}+k_{1} \varepsilon\right)\left|y_{1}-y_{2}\right| .
\end{aligned}
$$

This shows that, for $|\varepsilon| \leqq \bar{\varepsilon}_{0}, \mathscr{F}$ maps $S$ into itself, $\mathscr{F}: S \rightarrow S$, and $S$ is a convex closed compact subset of a Banach space. By SCHAUDER's fixed point theorem $\mathscr{F}$ possesses at least one fixed element $w=\mathscr{F} w \in S, w=\left[u_{0}(x), v_{0}(y)\right]$, with $u_{0}(x)$, $v_{0}(y)$ satisfying relations (7) of Theorem I. Criterion I is thereby proved.

15. Example. The equation

$$
u_{x y}=\varepsilon(1-u)+\varepsilon^{2} g\left(x, y, u, u_{x}, u_{y}\right),
$$

where $g$ is periodic of period $2 \pi$ in $x$ and $y$, has a periodic solution $\varphi(x, y)$ of the same period,

$$
\varphi(x, y)=1+O(\varepsilon) .
$$


The analogous equation

$$
u_{x y}=\varepsilon\left(\sin x-\cos x-\sin y+\cos y+u+u_{x}+u_{y}\right)+\varepsilon^{2} g\left(x, y, u, u_{x}, u_{y}\right)
$$

with $g$ as above has a periodic solution

$$
\varphi=\cos x-\cos y+O(\varepsilon) .
$$

16. Another Equation Containing a Small Parameter. Let us consider the differential equation

$$
\begin{aligned}
u_{x y} & =f\left(x, y, u, u_{x}, u_{y}\right), \\
f & =\psi(x, y)+C u+\psi_{1}(y) u_{x}+\psi_{2}(x) u_{y}+\varepsilon g\left(x, y, u, u_{x}, u_{y}\right),
\end{aligned}
$$

where $\varepsilon$ is a small parameter, and $\psi, \psi_{1}, \psi_{2}, g$ are as in Section 12. We assume here that, for $\varepsilon=0$, equation (63) possesses a known periodic solution of period $T$ in $x$ and $y$, of the form

$$
\varphi_{0}(x, y)=u_{0}(x)+v_{0}(y),
$$

where $u_{0}(x), v_{0}(y)$ have Fourier series (47), and hence

$$
\psi(x, y)=-C u_{0}(x)-C v_{0}(y)-\psi_{1}(y) u_{0}^{\prime}(x)-\psi_{2}(x) v_{0}^{\prime}(y) .
$$

Under the hypotheses below, we shall prove that for $|\varepsilon| \neq 0$ sufficiently small, (63) possesses a solution $\varphi(x, y)=\varphi_{0}(x, y)+O(\varepsilon)$ which is periodic of period $T$ in $x$ and $y$.

17. Criterion II. If the function $f$ defined in (63) for all $|\varepsilon| \leqq \varepsilon_{0}$ satisfies all conditions of Theorems I, II, III with given constants $T, N, N_{1}, N_{2}, L, M_{1}$, $M_{2}, M_{3}, b_{0}, b_{1}, b_{2}$ and in addition $f$ is Lipschitzian with respect to $x$ and $y$ in $R$, if $C \neq 0$, if (63) possesses for $\varepsilon=0$ a solution $\varphi_{0}(x, y)=U(x)+V(y)$ with $U, V$ periodic of period $T$, if the functions $U(x), V(y), \kappa_{1}(x), \kappa_{2}(y)$ are of class $C^{1}$ with Lipschitzian first derivative, and

$$
\begin{gathered}
|U(0)| \leqq N_{0}<N, \quad\left|U\left(x_{1}\right)-U\left(x_{2}\right)\right| \leqq N_{10}\left|x_{1}-x_{2}\right|, \quad N_{10}<N_{1}, \\
\left|V\left(y_{1}\right)-V\left(y_{2}\right)\right| \leqq N_{20}\left|y_{1}-y_{2}\right|, \quad N_{20}<N_{2},
\end{gathered}
$$

then there is some $\bar{\varepsilon}_{0}, 0<\bar{\varepsilon}_{0} \leqq \varepsilon_{0}$, such that equation (63) for all $|\varepsilon| \leqq \bar{\varepsilon}_{0}$, possesses at least one periodic solution $\varphi(x, y)$ of period $T$ in $x$ and $y$, which is Lipschitzian in $E_{2}$ together with $\varphi_{x}, \varphi_{y}, \varphi_{x y}$ :

$$
\varphi_{x y}=f\left(x, y, \varphi, \varphi_{x}, \varphi_{y}\right), \quad \varphi(x+T, y)=\varphi(x, y)=\varphi(x, y+T) .
$$

Moreover, the periodic functions $u_{0}(x)=\varphi(x, 0)=\varphi(x, T), v_{0}(y)=\varphi(0, y)-\varphi(0,0)$ $=\varphi(T, y)-\varphi(T, 0)$ satisfy relations $(7)$ of Theorem $\mathrm{I}$.

18. Proof of Criterion II. As in Section 12 let us apply formally the method of successive approximations of Section 10 to equation (63) with arbitrary initial values $u_{0}(x), v_{0}(y)$. Then the quadruple $[\varphi, m, n, \mu]$, the solution of the modified problem for equation (63), is given by

$$
\begin{aligned}
\varphi(x, y, \varepsilon) & =\varphi_{0}(x, y)+\tilde{\varphi}(x, y, \varepsilon), & m(y, \varepsilon) & =m_{0}(y)+\tilde{m}(y, \varepsilon), \\
n(x, \varepsilon) & =n_{0}(x)+\tilde{n}(x, \varepsilon), & \mu(\varepsilon) & =\mu_{0}+\tilde{\mu}(\varepsilon),
\end{aligned}
$$


where $\tilde{\varphi}, \tilde{m}, \tilde{n}, \tilde{\mu}=0(1)$ uniformly as $\varepsilon \rightarrow 0$, and $\varphi_{0}, m_{0}, n_{0}, \mu_{0}$ are given by formulas (48). In addition, we know that for $u_{0}(x) \equiv U(x), v_{0}(y) \equiv V(y)$ we have $\mu_{0}=0, m_{0}(y) \equiv 0, n_{0}(x) \equiv 0$. We can now repeat with obvious variants the argument of the proof of Criterion I.

19. Examples. The equation

$$
u_{x y}=-1+u+\psi_{1}(y) u_{x}+\psi_{2}(x) u_{y}+\varepsilon g\left(x, y, u, u_{x}, u_{y}\right)
$$

for $\varepsilon=0$ has the obvious solution $u=1$. Since $C \neq 0$, the same equation has a periodic solution $\varphi$ of period $T$ in $x$ and $y$ for all $|\varepsilon|$ sufficiently small.

Analogously, the equation

$$
u_{x y}=-\cos x+\sin x+\cos y-\sin y+u+u_{x}+u_{y}+\varepsilon g\left(x, y, u, u_{x}, u_{y}\right)
$$

has, for $\varepsilon=0$, the obvious solution $u=\cos x-\cos y$. Since $C \neq 0$, the same equation has a periodic solution of period $2 \pi$ in $x$ and $y$ for every $|\varepsilon| \neq 0$ sufficiently small.

20. Application to the Wave Equation. Let us consider the differential equation

$$
u_{t t}-u_{\xi \xi}=f\left(t, \xi, u, u_{t}, u_{\xi}\right),
$$

where $f$ is periodic in $t$ and $\xi$ of period $T$. Then the transformation

$$
t=x+y, \quad \xi=x-y, \quad x=2^{-1}(t+\xi), \quad y=2^{-1}(t-\xi),
$$

changes (64) into

where

$$
u_{x y}=F\left(x, y, u, u_{x}, u_{y}\right)
$$

$$
F=f\left(x+y, x-y, u, 2^{-1} u_{x}+2^{-1} u_{y}, 2^{-1} u_{x}-2^{-1} u_{y}\right),
$$

and $F$ is periodic of period $T$ in $x$ and $y$. Theorems I, II, III and the criteria should now be applied to (66). Other transformations beside (65) can be used.

As an example, let us consider the equation

$$
\begin{aligned}
u_{t t}-u_{\xi \xi}= & \varepsilon\left[\lambda(t, \xi)+C_{0} u+\lambda_{1}(t, \xi) u_{t}+\lambda_{2}(t, \xi) u_{\xi}\right]+\varepsilon^{2} g\left(t, \xi, u, u_{t}, u_{\xi}\right), \\
\lambda(t, \xi)= & A_{0}+B_{1} \cos 2 t+C_{1} \sin 2 t+B_{2} \cos 2 \xi+C_{2} \sin 2 \xi+ \\
& +D_{1} \cos (t+\xi)+E_{1} \sin (t+\xi)+D_{2} \cos (t-\xi)+E_{2} \sin (t-\xi), \\
\lambda_{1}(t, \xi)= & A+B \cos (t+\xi)+C \sin (t+\xi)+D \cos (t-\xi)+E \sin (t-\xi), \\
\lambda_{2}(t, \xi)= & A^{\prime}-B \cos (t+\xi)-C \sin (t+\xi)+D \cos (t-\xi)+E \sin (t-\xi),
\end{aligned}
$$

where $A_{0}, B, \ldots, E$ are constants, $C_{0} \neq 0$, and $g$ is of period $\pi$ in $t$ and $\xi$. By the transformation

$$
t=2^{-1}(x+y), \quad \xi=2^{-1}(x-y), \quad x=t+\xi, \quad y=t-\xi,
$$

equation (67) is changed into

$$
\begin{aligned}
u_{x y}= & \varepsilon\left[\psi(x, y)+4^{-1} C_{0} u+\psi_{1}(y) u_{x}+\psi_{2}(x) u_{y}\right]+ \\
& +\varepsilon^{2} g\left(2^{-1} x+2^{-1} y, 2^{-1} x-2^{-1} y, u, u_{x}+u_{y}, u_{x}-u_{y}\right),
\end{aligned}
$$


where the second member has period $2 \pi$ in $x$ and $y$, and

$$
\begin{aligned}
& \psi(x, y)=a_{00}+a_{10} \cos x+c_{01} \sin x+a_{01} \cos y+b_{01} \sin y+a_{11} \cos x \cos y+ \\
& +b_{11} \cos x \sin y+c_{11} \sin x \cos y+d_{11} \sin x \sin y, \\
& \psi_{1}(y)=e_{0}+e_{1} \cos y+f_{1} \sin y, \quad \psi_{2}(x)=g_{0}+g_{1} \cos x+h_{1} \sin y, \\
& 4 a_{00}=A_{0}, \quad 4 a_{10}=D_{1}, \quad 4 c_{01}=E_{1}, \quad 4 a_{01}=D_{2}, \quad 4 b_{01}=E_{2}, \\
& 4 a_{11}=B_{1}+B_{2}, \quad 4 b_{11}=C_{1}-C_{2}, \quad 4 c_{11}=C_{1}+C_{2}, \quad 4 d_{11}=-B_{1}+B_{2}, \\
& 4 e_{0}=A+A^{\prime} \text {, } \\
& 2 e_{1}=D, \quad 2 f_{1}=E, \quad 4 g_{0}=A-A^{\prime}, \quad 2 g_{1}=B, \quad 2 h_{1}=C .
\end{aligned}
$$

By Criterion I we conclude that if $C_{0} \neq 0$ and $|\varepsilon|$ sufficiently small, then equation (70) has at least a periodic solution $\varphi(x, y, \varepsilon)$ of period $2 \pi$ in $x$ and $y$, and then equation (67) has at least a solution

$$
u(t, \xi, \varepsilon)=\varphi(t+\xi, t-\zeta, \varepsilon),
$$

also of period $2 \pi$ in $t$ and $\xi$.

21. Another Example. Let us consider the differential equation

$$
u_{t t}-u_{\xi \xi}=\lambda(t, \xi)+C_{0} u+\lambda_{1}(t, \xi) u_{t}+\lambda_{2}(t, \xi) u_{\xi}+\varepsilon g\left(t, \xi, u, u_{t}, u_{\xi}\right),
$$

where $\lambda, \lambda_{1}, \lambda_{2}$ are given by (68) and again $C_{0} \neq 0$. By the same transformation (69) equation (72) is changed into

$$
\begin{aligned}
u_{x y}= & \psi(x, y)+4^{-1} C_{0} u+\psi_{1}(y) u_{x}+\psi_{2}(x) u_{y}+ \\
& +\varepsilon g\left(2^{-1} x+2^{-1} y, 2^{-1} x-2^{-1} y, u, u_{x}+u_{y}, u_{x}-u_{y}\right),
\end{aligned}
$$

where $\psi, \psi_{1}, \psi_{2}$ are given by formulas (71). It is immediately seen that (73) for $\varepsilon=0$ has a solution of the form

if and only if

$$
\begin{aligned}
u(x, y) & =U(x)+V(y), \quad U(x)=\alpha_{0}+\alpha_{1} \cos x+\beta_{1} \sin x, \\
V(y) & =\gamma_{1} \cos y+\delta_{1} \sin y
\end{aligned}
$$

$$
\begin{aligned}
B_{1}= & \Delta_{1} C_{0}\left(E D_{1}+D E_{1}\right)+\Delta_{1}\left(A+A^{\prime}\right)\left(D D_{1}-E E_{1}\right)+ \\
& +\Delta_{2} C_{0}\left(C D_{2}+B E_{2}\right)+\Delta_{2}\left(A-A^{\prime}\right)\left(B D_{2}-C E_{2}\right), \\
B_{2}= & \Delta_{1} C_{0}\left(-E D_{1}+D E_{1}\right)+\Delta_{1}\left(A+A^{\prime}\right)\left(D D_{1}-E E_{1}\right)+ \\
& +\Delta_{2} C_{0}\left(-C D_{2}+B E_{2}\right)+\Delta_{2}\left(A-A^{\prime}\right)\left(B D_{2}+C E_{2}\right), \\
C_{1}= & \Delta_{1} C_{0}\left(-D D_{1}+E E_{1}\right)+\Delta_{1}\left(A+A^{\prime}\right)\left(E D_{1}+D E_{1}\right)+ \\
& +\Delta_{2} C_{0}\left(-B D_{2}+C E_{2}\right)+\Delta_{2}\left(A-A^{\prime}\right)\left(C D_{2}+B E_{2}\right), \\
C_{2}= & \Delta_{1} C_{0}\left(-D D_{1}-E E_{1}\right)+\Delta_{1}\left(A+A^{\prime}\right)\left(-E D_{1}+D E_{1}\right)+ \\
& +\Delta_{2} C_{0}\left(B D_{2}+C E_{2}\right)+\Delta_{2}\left(A-A^{\prime}\right)\left(C D_{2}-B E_{2}\right), \\
\Delta_{1}= & \left(C_{0}^{2}+\left(A+A^{\prime}\right)^{2}\right)^{-1}, \quad \Delta_{2}=\left(C_{0}^{2}+\left(A-A^{\prime}\right)^{2}\right)^{-1} .
\end{aligned}
$$


In this situation then

$\alpha_{1}=\Delta_{1}\left(-C_{0} D_{1}+\left(A+A^{\prime}\right) E_{1}\right), \quad \beta_{1}=\Delta_{1}\left(-C_{0} E_{1}-\left(A+A^{\prime}\right) D_{1}\right)$,

$\gamma_{1}=\Delta_{2}\left(-C_{0} D_{2}+\left(A-A^{\prime}\right) E_{2}\right), \quad \delta_{1}=\Delta_{2}\left(-C_{0} E_{2}-\left(A-A^{\prime}\right) D_{2}\right), \quad \alpha_{0}=-C_{0}^{-1} A_{0}$.

By Criterion II we conclude that if $C_{0} \neq 0,|\varepsilon|$ sufficiently small, and relations (74) hold, then equation (73) has a periodic solution $\varphi(x, y)$ of period $2 \pi$ in $x$ and $y$, and (72) has a solution

$$
u(t, \xi, \varepsilon)=\varphi(t+\xi, t-\xi, \varepsilon)
$$

also of period $2 \pi$ in $t$ and $\xi$.

For instance, for the equation

$$
\begin{aligned}
u_{t t}-u_{\xi \xi}= & D_{1} \cos (t+\xi)+E_{1} \sin (t+\xi)+D_{2} \cos (t-\xi)+ \\
& +E_{2} \sin (t-\xi)+u+u_{t}+\varepsilon g\left(t, \xi, u, u_{t}, u_{\xi}\right),
\end{aligned}
$$

where $D_{1}, E_{1}, D_{2}, E_{2}$ are arbitrary constants and $g$ periodic of period $\pi$ in $t$ and $\xi$, we see that relations (74) are all satisfied with

$$
\begin{array}{cc}
B_{1}=C_{1}=B_{2}=C_{2}=0, & B=C=D=E=0, \quad A_{0}=0, \quad C_{0}=1, \quad A=1, \\
& A^{\prime}=0, \quad \Delta_{1}=\Delta_{2}=2^{-1} .
\end{array}
$$

The corresponding equation (73) is

$$
4 u_{x y}=D_{1} \cos x+E_{1} \sin x+D_{2} \cos y+E_{2} \sin y+u+u_{x}+u_{y}+\varepsilon g .
$$

For $\varepsilon=0$ this equation has the periodic solution $\varphi_{0}(x, y)$ of period $2 \pi$ in $x, y$ given by

$$
-2 \varphi_{0}(x, y)=\left(D_{1}-E_{1}\right) \cos x+\left(D_{1}+E_{1}\right) \sin x+\left(D_{2}-E_{2}\right) \cos y+\left(D_{2}+E_{2}\right) \sin y,
$$

and hence (75) for $\varepsilon=0$ has the periodic solution

$$
\begin{aligned}
u_{0}(t, \xi)= & -2^{-1}\left(D_{1}-E_{1}\right) \cos (t+\xi)-2^{-1}\left(D_{1}+E_{1}\right) \sin (t+\xi)- \\
& -2^{-1}\left(D_{2}-E_{2}\right) \cos (t-\xi)-2^{-1}\left(D_{2}+E_{2}\right) \sin (t-\xi) .
\end{aligned}
$$

Thus, for all $|\varepsilon|$ sufficiently small equation (75) has a periodic solution of period $2 \pi$ in $t$ and $\xi$ of the form $u(t, \xi)=u_{0}(t, \xi)+O(\varepsilon)$.

22. Remark. In the autonomous case, that is, when $f$ does not depend on $x$ and $y$, then $\psi=a_{00}, \psi_{1}=e_{0}, \psi_{2}=g_{0}, g=g\left(u, u_{x}, u_{y}\right)$. It is easy to verify that the periodic solution $\varphi$ of equation (46), whose existence is proved by Criterion I, is a constant.

Concerning Criterion II, let us note first that equation (63) in the autonomous case reduces to

$$
u_{x y}=a_{00}+C u+e_{0} u_{x}+g_{0} u_{y}+\varepsilon g\left(u, u_{x}, u_{y}\right) .
$$

Under the hypothesis of Criterion II we have $C \neq 0$ and

$$
\left|C\left(z_{1}-z_{2}\right)+\varepsilon g\left(z_{1}, p, q\right)-\varepsilon g\left(z_{2}, p, q\right)\right| \leqq b_{0}\left|z_{1}-z_{2}\right|
$$

for all $z_{1}, z_{2}, p, q, \varepsilon$ with $\left|z_{1}\right|,\left|z_{2}\right| \leqq M_{1},|p| \leqq M_{2},|q| \leqq M_{3},|\varepsilon| \leqq \varepsilon_{0}$. We may well assume $b_{0}>0$. From (77) we deduce first, taking $\varepsilon=0,|C|<b_{0}$, and then, taking $\varepsilon=\varepsilon_{0},\left|g\left(z_{1}, p, q\right)-g\left(z_{2}, p, q\right)\right| \leqq\left(b_{0}+|C|\right) \varepsilon_{0}^{-1}\left|z_{1}-z_{2}\right| \leqq 2 b_{0} \varepsilon_{0}^{-1}\left|z_{1}-z_{2}\right|$, 
and hence $g$ is uniformly Lipschitzian with constant $2 b_{0} \varepsilon_{0}^{-1}$. Then, for $|\varepsilon| \leqq \varepsilon_{1}=$ $\min \left[\varepsilon_{0}, \varepsilon_{0}|C| / 4 b_{0}\right]$, we see that $\varepsilon g(z, p, q)$ is uniformly Lipschitzian in $z$ with constant $|C| / 2$. Then for $|\varepsilon| \leqq \varepsilon_{1}$ and $C \neq 0$, the expression $a_{00}+C z+\varepsilon g(z, 0,0)$ is monotone, namely strictly increasing or strictly decreasing as $C z$. Note that, for $\gamma_{0}=C^{-1}\left|a_{00}\right|<M_{1}$, there is a unique $\gamma_{0}=\gamma(0)=C^{-1} a_{00}$ such that $\gamma=\gamma_{0}$, $\varepsilon=0$ satisfy the equation

$$
a_{00}+C \gamma+\varepsilon g(\gamma, 0,0)=0,
$$

and thus there is some $\varepsilon_{2}, 0<\varepsilon_{2} \leqq \varepsilon_{1}$, and a constant $\gamma=\gamma(\varepsilon)$ such that (78) is satisfied for $|\varepsilon| \leqq \varepsilon_{2}$. Then equation (76) is satisfied for $\varepsilon=0$ by the constant function $\varphi(x, y)=U(x)+V(y)=\gamma_{0}$, with $U=\gamma_{0}, V=0$ and equation (76) is satisfied for $|\varepsilon| \leqq \varepsilon_{2}$ by the constant function $\varphi(x, y)=\gamma(\varepsilon)$.

The research reported here was supported in part by U.S. National Science Foundation grant G-57 at the University of Michigan.

\section{Bibliography}

[1] Artem'ev, N. A., Periodic solutions of a class of partial differential equations. Izv. Akad. Nauk, SSSR Ser. Mat. 1 (1937) [Russian].

[2] Cesari, L., (a) Periodic solutions of hyperbolic partial differential equations. Intern. Symp. of Nonlinear Differential Equations and Nonlinear Mechanics (Colorado Springs, 1961). Academic Press 1963; 33-57; Intern. Symp. on Nonlinear Oscillations (Kiev. 1961). Izdat. Akad. Nauk, SSSR 2, 440-457 (1963); - (b) A criterion for the existence in a strip of periodic solutions of hyperbolic partial differential equations (to appear).

[3] Fleischman, B. A., \& F. A. Ficken, Initial value and time periodic solutions for a nonlinear wave equation. Comm. Pure Appl. Math. 10, 331-356 (1957).

[4] KARP, V. N., (a) Application of the wave-region method to the problem of forced nonlinear periodic vibrations of a string. Izv. Vyss. Vc. Zaved. Mat. 6 (25), 51-59 (1961) [Russian]; - (b) On periodic solutions of nonlinear hyperbolic equations. Dokl. Akad. Nauk. Uzbek. SSR 5 (1953) [Russian].

[5] Mitryakov, A. P., (a) On periodic solutions of the nonlinear hyperbolic equation. Trudy Inst. Mat. Meh. Akad. Nauk Uzbek. SSR 7, 137-149 (1949) [Russian]; - (b) On solutions of infinite systems of nonlinear integral and integro-differential equations. Trudy Uzbek. Gosudarstv. Univ. 37 (1948) [Russian].

[6] Prodi, C., Soluzioni periodiche di equazioni alle derivate parziali di tipo iperbolico nonlineari. Annali Mat. Pura. Appl. 42, 25-49 (1956).

[7] Solov'Ev, P. V., Some remarks on periodic solutions of the nonlinear equation of hyperbolic type. Izv. Akad. Nauk. SSSR, Ser. Mat. 2, 150-164 (1939) [Russian].

[8] Sokolov, C. T., On periodic solutions of a class of partial differential equations. Dokl. Akad. Nauk. Uzbek. SSR 12, 3-7 (1953) [Russian].

[9] Vejvoda, O., Nonlinear boundary-value problems for differential equations. Proc. Conference Differential Equations and Their Applications (Prague 1962). Prague: Publishing House Czechosl. Acad. Sci. 1963, 199-215.

[10] ZabotinsKII, M. E., On periodic solutions of nonlinear partial differential equations. Dokl. Akad. Nauk SSSR (N.S.) 56, 469-472 (1947) [Russian]. 